\title{
ESTIMATIVA DO COEFICIENTE DE DILATAÇÃO VOLUMÉTRICA DO SEBO BOVINO REFINADO DERRETIDO A PARTIR DA APLICAÇÃO DA ANÁLISE DE REGRESSÃO LINEAR
}

César Augusto CANCIAM*

\begin{abstract}
*Mestre em Engenharia Química, professor lotado no Departamento Acadêmico de Engenharia Química da Universidade Tecnológica Federal do Paraná - Câmpus Ponta Grossa, canciam@utfpr.edu.br
\end{abstract}

Recebido em: 30/05/2014 - Aprovado em: 29/09/2014 - Disponibilizado em: 15/12/2014

\begin{abstract}
RESUMO:
O coeficiente de dilatação volumétrica está relacionado com a resposta dos materiais à aplicação de calor. E fornece uma medida da variação da massa específica em resposta a uma mudança de temperatura, sob condição de pressão constante. O presente trabalho teve por objetivo estimar o coeficiente de dilatação volumétrica do sebo bovino refinado derretido a partir das modelagens matemáticas propostas por ALVARADO (1995) e CANCIAM (2005). Para tanto, foi realizada uma análise de regressão linear de dados da massa específica em função da temperatura. Os valores obtidos para o coeficiente de dilatação volumétrica foram de $8,2320 \times 10^{-4}{ }^{\circ} \mathrm{C}^{-1}$ (de acordo com a metodologia proposta por ALVARADO, 1995) e $7,8043 \times 10^{-4}{ }^{\circ} \mathrm{C}^{-1}$ (segundo a metodologia proposta por CANCIAM, 2005). Nas duas metodologias, os coeficientes de correlação foram próximos da unidade. Na literatura, não foram encontrados valores experimentais para o coeficiente de dilatação volumétrica do sebo bovino refinado derretido. Os resultados sugerem que o coeficiente de dilatação volumétrica do sebo bovino derretido está próximo ao coeficiente de dilatação volumétrica do óleo de soja refinado.
\end{abstract}

PALAVRAS-CHAVE: Dilatação. Sebo bovino derretido. Estimativa. Regressão linear.

\section{ESTIMATE OF THE COEFFICIENT OF VOLUMETRIC DILATATION OF REFINED BEEF TALLOW MELTED BY APPLICATION OF LINEAR REGRESSION ANALYSIS}

\begin{abstract}
:
The coefficient of volumetric dilatation is associated with response of materials to the application of heat. This coefficient provides a measure of specific weight variation in response to temperature variation, keeping the pressure constant. This study aimed to estimate the coefficient of volumetric dilatation of refined beef tallow melted from the mathematical models proposed by ALVARADO (1995) and CANCIAM (2005). For this purpose, it was performed a linear regression analysis of the data of specific weight in a function of temperature. The values obtained for the coefficient of volumetric dilatation were $8.2320 \mathrm{x} 10^{-4}{ }^{\circ} \mathrm{C}^{-1}$ (according to the methodology proposed by ALVARADO, 1995) and $7.8043 \times 10^{-4} \circ \mathrm{C}^{-1}$ (according to the methodology proposed by CANCIAM, 2005). In both methodologies, the correlation coefficients were close to unity. In the literature, not found experimental values for the coefficient of volumetric dilatation of refined beef tallow melted. The results suggest that the coefficient of volumetric dilatation of refined beef tallow melted is near the coefficient of volumetric dilatation of refined soybean oil.
\end{abstract}

KEYWORDS: Dilatation. Beef tallow melted. Estimate. Linear regression. 


\section{INTRODUÇÃO}

A carne é considerada o principal produto do abate bovino. Como subprodutos destacam-se pele, ossos, colágeno e sebo. $\mathrm{O}$ sebo bovino vem sendo utilizado como matéria-prima para as indústrias de higiene e limpeza e na produção de biodiesel (MARTINS et al., 2011).

De acordo com MARTINS e CARNEIRO (2013), a soja é utilizada em $78,48 \%$ da produção brasileira de biodiesel. $\mathrm{O}$ sebo bovino ocupa a segunda posição, com uma participação em torno de $17 \%$.

O sebo bovino vem sendo utilizado como suplemento energético pelas indústrias de ração (FERREIRA, 2004), nas indústrias de cosméticos, tintas, vernizes e lubrificantes; na conservação do couro, no uso veterinário e como matéria-prima na produção de estearina (DÍAZ, 2012).

Em função do baixo preço, sebo bovino vem sendo utilizado na queima em caldeiras de pequeno porte. Segundo ANDRADE FILHO (2007), no Brasil é proibido o uso de sebo para o consumo humano.

Sebo bovino é constituído por triglicerídeos, que à temperatura ambiente, encontram-se pastosos. A $40^{\circ} \mathrm{C}$, o sebo bovino é fundido, tornando-se líquido. A temperatura de fusão do sebo bovino está relacionada com a sua composição em termos de ácidos graxos. De acordo com ANDRADE FILHO (2007), mais de 50\% da estrutura molecular dos triglicerídeos é saturada. Os ácidos graxos encontrados são oléico (45\%), palmítico (30\%), esteárico (20 a 25\%), mirístico (3 a $7 \%$ ), linoléico (1 a 3\%) e palmitoléico (1 a 3\%) (ANDRADE FILHO, 2007).

A dilatação de um material devido à elevação da temperatura é uma consequência do aumento da sua energia interna, que determina uma maior amplitude das vibrações moleculares e, portanto, uma maior distância média entre os constituintes moleculares (FRADE, 2010).

O coeficiente de dilatação volumétrica $(\gamma)$, também chamado de coeficiente de expansão térmica, é uma propriedade volumétrica derivada da massa específica $(\rho)$ e está relacionada com a resposta de um material à aplicação de calor (AMORIM, 2007; CALLISTER, 2008).

Segundo JERÔNIMO et al. (2012), a dilatação volumétrica é variável e tende a manter uma relação direta com a composição química.

SANTOS e VIEIRA (2010) comentam que a partir do conhecimento do coeficiente de dilatação volumétrica é possível estimar outras propriedades físico-químicas e avaliar os impactos nos sistemas de medição 
volumétrica devidos à mudança de temperatura.

O objetivo deste trabalho foi estimar o coeficiente de dilatação volumétrica do sebo bovino refinado derretido através da modelagem matemática de dados da massa específica em função da temperatura. Os modelos matemáticos utilizados foram os de ALVARADO (1995) e CANCIAM (2005).

\section{MATERIAIS E MÉTODOS}

O modelo matemático proposto por ALVARADO (1995) considera a análise de regressão linear de dados de $\frac{1}{\rho}$ em função da temperatura $(T)$ para a obtenção do coeficiente de dilatação volumétrica $(\gamma)$. A Equação 1 relaciona o modelo matemático proposto por ALVARADO (1995).

$$
\frac{1}{\rho}=\left(\frac{1}{\rho^{*}}\right)+\left(\frac{\gamma}{\rho^{*}}\right) \cdot T
$$

Já o modelo matemático proposto por CANCIAM (2005) considera a análise de regressão linear de dados de $\ln \left(\frac{\rho_{0}}{\rho}\right)$ em função de $\left(T-T_{0}\right)$ para a obtenção do coeficiente de dilatação volumétrica $(\gamma)$. A
Equação 2 relaciona o modelo matemático proposto por CANCIAM (2005).

$$
\ln \left(\frac{\rho_{0}}{\rho}\right)=\gamma \cdot\left(T-T_{0}\right)
$$

Em que $\rho_{0}$ corresponde à massa específica na temperatura $T_{0}$.

A Tabela 1 relaciona os dados da massa específica em função da temperatura para o sebo bovino refinado derretido. A faixa de temperatura considerada variou de 50 a $80^{\circ} \mathrm{C}$.

Os dados da massa específica do sebo bovino refinado derretido foram adaptados do trabalho de CAVALCANTI et al. (2009).

A partir dos dados da Tabela 1 e das Equações 1 e 2 foi possível realizar a análise de regressão linear.

Tabela 1. Efeito da temperatura na massa específica do sebo bovino refinado

\begin{tabular}{cc}
\hline Temperatura $\left({ }^{\circ} \mathrm{C}\right)$ & Massa específica $\left(\mathrm{kg} . \mathrm{m}^{-3}\right)$ \\
\hline 50 & 880 \\
52 & $878,65^{*}$ \\
54 & $877,26^{*}$ \\
56 & $875,86^{*}$ \\
58 & $874,43^{*}$ \\
60 & 873 \\
62 & $871,57^{*}$ \\
64 & $870,14^{*}$ \\
66 & $868,74^{*}$ \\
68 & $867,35^{*}$ \\
70 & 866 \\
72 & $864,69^{*}$ \\
74 & $863,42^{*}$ \\
76 & $862,22^{*}$ \\
78 & $861,07^{*}$ \\
80 & 860 \\
\hline
\end{tabular}

Fonte: Adaptado de CAVALCANTI et al. (2009).

* - Valores obtidos pelo método de interpolação de Lagrange. 
Cada uma das análises de regressão linear foi realizada utilizando um programa específico disponível na calculadora CASIO FX-850P Scientific Library 116.

Para o método da interpolação de Lagrange também foi realizado utilizando um programa específico disponível na mesma calculadora.

Nos cálculos para o modelo matemático de CANCIAM (2005), considerou-se a temperatura $T_{0}$ igual a $50^{\circ} \mathrm{C}$.

\section{RESULTADOS E DISCUSSÃO}

A Figura 1 ilustra o diagrama de dispersão dos valores de $\frac{1}{\rho}$ em função da temperatura, considerando o modelo matemático proposto por ALVARADO (1995).

Figura 1: Diagrama de dispersão dos valores de $\frac{1}{\rho}$ em função da temperatura.

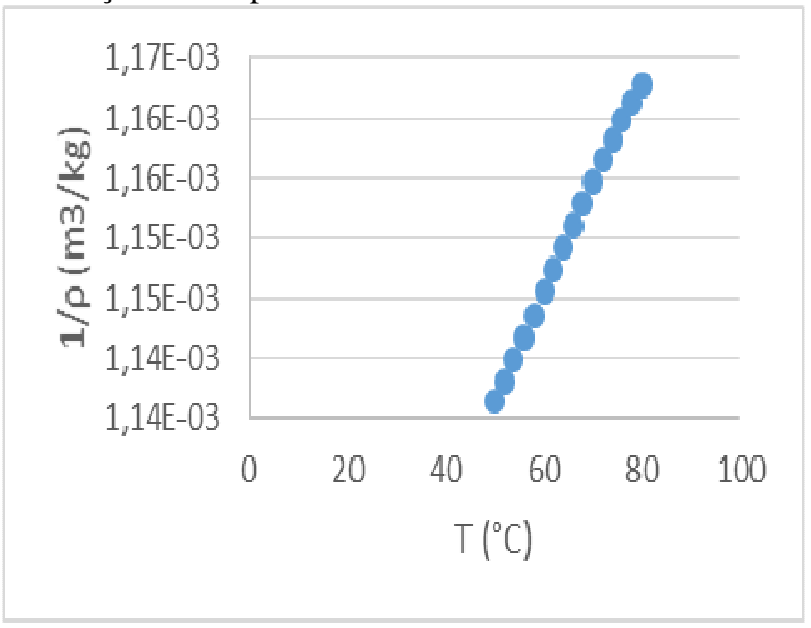

Fonte: Autor.
Enquanto que a Figura 2 ilustra o diagrama de dispersão dos valores de $\ln \left(\frac{\rho_{o}}{\rho}\right)$ em função de $\left(T-T_{0}\right)$, considerando o modelo matemático proposto por CANCIAM (2005).

A Tabela 2 indica os resultados obtidos pela análise de regressão linear, considerando o modelo de ALVARADO (1995).

Figura 2: Diagrama de dispersão dos valores de $\ln \left(\frac{\rho_{o}}{\rho}\right)$ em função de $\left(T-T_{0}\right)$

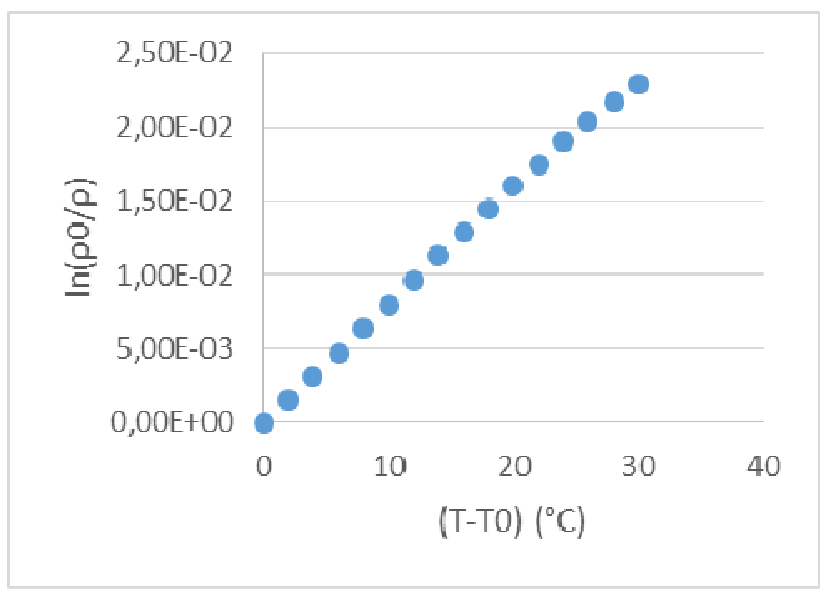

Fonte: Autor.

Tabela 2. Resultados da análise linear considerando o modelo de ALVARADO (1995)

\begin{tabular}{cc} 
modelo de ALVARADO (1995) \\
\hline Parâmetros & Valores \\
\hline$\left(\frac{1}{\rho^{*}}\right)$ & $1,09 \times 10^{-3} \mathrm{~m}^{3} \cdot \mathrm{kg}^{-1}$ \\
$\left(\frac{\gamma}{\rho^{*}}\right)$ & $8,9729 \times 10^{-7} \mathrm{~m}^{3} \cdot{ }^{\circ} \mathrm{C} \cdot \mathrm{kg}^{-1}$ \\
$R^{2}$ & 0,9991 \\
\hline
\end{tabular}

Fonte: Autor. 
Para o modelo proposto por ALVARADO (1995), com base na Tabela 2, o parâmetro $\rho^{*}$ e o coeficiente de dilatação volumétrica $(\gamma)$ equivalem, respectivamente a, $917,4312 \mathrm{~kg} \cdot \mathrm{m}^{-3}$ e $8,2320 \times 10^{-4}{ }^{\circ} \mathrm{C}^{-1}$.

A Tabela 3 indica os resultados obtidos pela análise de regressão linear, considerando o modelo de CANCIAM (2005).

Tabela 3. Resultados da análise linear considerando o modelo de CANCIAM (2005)

\begin{tabular}{cc}
\hline Parâmetros & Valores \\
\hline Intercepto & $1,5055 \times 10^{-4}$ \\
$\gamma$ & $7,8043 \times 10^{-4}{ }^{\circ} \mathrm{C}^{-1}$ \\
$R^{2}$ & 0,9989 \\
\hline
\end{tabular}

Fonte: Autor

TRIOLA (2008) comenta que o coeficiente de correlação $\left(R^{2}\right)$ mede o grau de relacionamento linear entre os valores emparelhados das variáveis dependente e independente. Ou seja, quanto mais próximo for esse coeficiente da unidade, melhor é a qualidade do ajuste da função em relação aos pontos do diagrama de dispersão.

LIRA (2004) acrescenta que para valores do coeficiente de correlação maiores ou iguais a 0,90 e menores que 1 , a correlação linear é classificada como muito forte.

Com base nas Tabelas 2 e 3, pode-se verificar que para os modelos matemáticos estudados, a correlação linear é classificada como muito forte.
SKOOG et al. (2006) comentam que a partir de um modelo de regressão linear, pode-se empregar a análise de variância (ANOVA) para verificar quanto a reta de regressão "explica" os valores observados que foram utilizados para o ajuste.

A Tabela 4 fornece a análise de variância (ANOVA) para o modelo proposto por ALVARADO (1995). Já a Tabela 5 fornece a análise de variância (ANOVA) para o modelo proposto por CANCIAM (2005).

Tabela 4 - ANOVA considerando o modelo de ALVARADO (1995)

\begin{tabular}{ccccc}
\hline $\begin{array}{c}\text { Fonte de } \\
\text { variação }\end{array}$ & GL & SQM & QM & Fcalc \\
\hline Regressão & 1 & $1,10 \times 10^{-9}$ & $1,10 \times 10^{-9}$ & 16585,86 \\
Resíduos & 14 & $9,24 \times 10^{-13}$ & $6,60 \times 10^{-14}$ & \\
Total & 15 & $1,10 \times 10^{-9}$ & & \\
\hline \multicolumn{5}{l}{ Fonte: Autor. }
\end{tabular}

Nas Tabelas 4 e 5, GL corresponde aos graus de liberdade; SQM, à soma dos quadrados médios; QM, ao quadrado médio e Fcal, ao valor de F calculado.

Tabela 5 - ANOVA considerando o modelo de CANCIAM (2005)

\begin{tabular}{ccccc}
\hline $\begin{array}{c}\text { Fonte de } \\
\text { variação }\end{array}$ & GL & SQM & QM & Fcalc \\
\hline Regressão & 1 & $8,28 \times 10^{-4}$ & $8,28 \times 10^{-4}$ & 14043,76 \\
Resíduos & 14 & $8,26 \times 10^{-7}$ & $5,60 \times 10^{-8}$ & \\
Total & 15 & $8,29 \times 10^{-4}$ & & \\
\hline
\end{tabular}

Fonte: Autor.

De acordo com TRIOLA (2008), no modelo de regressão linear simples a função 
do teste $\mathrm{F}$ é a de testar a significância do efeito de $\mathrm{X}$ sobre $\mathrm{Y}$, ou seja, testar a significância do efeito da temperatura sobre a massa específica.

Considerando um nível de significância de 5\%, o valor de Ftab (F tabelado) equivale a 4,60 (TRIOLA, 2008).

Como nas Tabelas 4 e 5 os valores de Fcal são maiores que o valor de Ftab, os resultados da ANOVA sugerem que a regressão linear como um todo faz sentido, ou seja, a variável explicativa temperatura influencia globalmente a variável explicada massa específica.

ALVARADO (1995), estudando a dilatação volumétrica de óleos vegetais entre 20 e $70^{\circ} \mathrm{C}$, obteve para o óleo de soja refinado um valor de $8,2 \times 10^{-4}{ }^{\circ} \mathrm{C}^{-1}$ para o coeficiente de dilatação volumétrica. Para o mesmo óleo, CANCIAM (2008), entre -20 e $80^{\circ} \mathrm{C}$, obteve um valor de $7,4676 \times 10^{-4} \quad{ }^{\circ} \mathrm{C}^{-1}$ para o coeficiente de dilatação volumétrica.

Comparando os valores encontrados neste trabalho, a diferença entre o coeficiente de dilatação volumétrica do sebo bovino refinado derretido e o coeficiente de dilatação volumétrica do óleo de soja refinado é de $3,2 \times 10^{-6}{ }^{\circ} \mathrm{C}^{-1}$ (segundo o modelo proposto por ALVARADO, 1995) e de $3,367 \times 10^{-5}{ }^{\circ} \mathrm{C}^{-1}$ (segundo o modelo proposto por CANCIAM, 2005).
Dessa forma, os resultados sugerem que o coeficiente de dilatação volumétrica do sebo bovino refinado derretido está próximo ao coeficiente de dilatação volumétrica do óleo de soja refinado, para os dois modelos matemáticos considerados neste trabalho.

Com relação ao valor experimental do coeficiente de dilatação volumétrica do sebo bovino refinado derretido, não foi encontrado nenhum valor na literatura.

O coeficiente de dilatação volumétrica mede a variação relativa do volume devido à mudança de temperatura, mantendo-se a pressão constante. Assim, por exemplo, se o coeficiente de dilatação volumétrica de um material é igual a $1,0 \times 10^{-2}{ }^{\circ} \mathrm{C}^{-1}$ significa que o acréscimo de 1 grau tem como resultado o aumento de volume em 1\% (NETZ e ORTEGA, 2008).

Dessa forma, seguindo a mesma linha de raciocínio, para cada 1 grau de acréscimo de temperatura, o aumento do volume do sebo bovino refinado derretido varia de 0,078\% (segundo a metodologia proposta por CANCIAM, 2005) e 0,082\% (de acordo com a metodologia proposta por ALVARADO, 1995).

Esses valores podem parecer pequenos, mas em comparação com a água, o aumento do volume do sebo bovino refinado derretido corresponde de 3,72 a 3,98 vezes mais que o aumento de volume de água. 
Nos cálculos acima citados, foi considerado que o coeficiente de dilatação volumétrica da água equivale a $2,07 \times 10^{-4}{ }^{\circ} \mathrm{C}^{-1}$ (CABRAL e LAGO, 2002).

\section{CONCLUSÃO}

A utilização de dados da massa específica em função da temperatura permitiu estimar o coeficiente de dilatação volumétrica do sebo bovino refinado derretido.

Os valores estimados para essa grandeza variaram de $7,8043 \times 10^{-4}{ }^{\circ} \mathrm{C}^{-1}$ (de acordo com a modelagem proposta por CANCIAM, 2005) a $8,2320 \times 10^{-4} \quad{ }^{\circ} \mathrm{C}^{-1}$ (segundo a modelagem proposta por ALVARADO, 1995).

Em comparação a outros óleos vegetais, os valores estimados neste trabalho encontram-se próximos ao coeficiente de dilatação volumétrica do óleo de soja refinado.

Em todas as análises de regressão linear, a correlação linear foi classificada como muito forte.

\section{REFERÊNCIAS}

ALVARADO, J. D. Propiedades mecânicas de aceites y grasas vegetales. Grasas y Aceites, v. 46, n. 4 - 5, p. 264-269, 1995.
AMORIM, J. A. Obtenção de propriedades físico-químicas de misturas de hidrocarbonetos em baixa e alta pressão visando a caracterização e a modelagem. Tese de Doutorado em Engenharia Química da Universidade Federal do Rio Grande do Norte, 2007. 165p.

ANDRADE FILHO, M. Aspectos técnicos e econômicos da produção do biodiesel: o caso do sebo bovino como matéria-prima. Dissertação de Mestrado em Regulação da Indústria de Energia da Universidade Salvador, 2007. 120p.

CABRAL, F.; LAGO, A. Física 2. Harbra, São Paulo, 2002. 516p.

CALLISTER, W. D. Ciência e Engenharia de Materiais: uma introdução. Livros Técnicos e Científicos, Rio de Janeiro, 2002. 450p.

CANCIAM, C. A. Predição do coeficiente de expansão térmica do óleo de algodão.

Publicatio UEPG - Ciências Exatas e da Terra, Ciências Agrárias e Engenharias, v. 11, n. 3, p. 27-31, 2005.

CANCIAM, C. A. Predição do coeficiente de expansão térmica do óleo de soja (Glicine max). Tecnológica, v. 17, p. 13-18, 2008. 
CAVALCANTI, L. A. P.; SCHULER, A. R. P.; DANTAS, C. C.; STRAGEVITCH, L.; ALMEIDA, Y. M. B.; SARMENTO, S. M. Caracterização reológica do sebo bovino refinado e do seu biodiesel. In: Congresso Brasileiro de Plantas Oleaginosas, Óleos, Gorduras e Biodiesel, 6, 2009, Montes Claros: Anais... Montes Claros: UFLA, 2009.

DÍAZ, G. C. Hidrólise e hidrogenação simultânea (óleo de soja e de sebo bovino) efeito do metal suportado. Tese de Doutorado em Tecnologia de Processos Químicos e Bioquímicos da Universidade Federal do Rio de Janeiro, 2012. 204p.

FERREIRA, A. F. Valor nutricional do óleo de soja, do sebo bovino e de suas combinações em rações para frangos de corte. Dissertação de Mestrado em Ciência Animal da Universidade Federal do Mato Grosso do Sul, 2004. 36p.

FRADE, J. M. C. B. C. Conformação automática de formas complexas em vidro de mesa. Tese de Doutorado em Ciências e Engenharia dos Materiais da Universidade de Aveiro, 2010. 327p.

JERÔNIMO, C. E. M.; BALBINO, C. P.; FERNANDES, H. G. Coeficiente de dilatação volumétrica determinados pela curva ASTM em frações de petróleo. Scientia Plena, v.8, n. 9, p. 1-8, 2012.

LIRA, S. A. Análise de correlação: abordagem teórica e de construção dos coeficientes com aplicação. Dissertação de Mestrado do Programa de Mestrado em Ciências da Universidade Federal do Paraná, 2004. 196p.

MARTINS, L. O. S.; CARNEIRO, R. A. F. O sebo bovino como insumo estratégico da cadeia de biodiesel: uma análise crítica. Bioenergia em revista: diálogos, v. 3, n.1, p. 32-44, 2013.

MARTINS, R.; NACHILUK, K.; BUENO, C. R. F.; FREITAS, S. M. O biodiesel de sebo bovino no Brasil. Informações Econômicas, v. 41, n. 5, p. 57-70, 2011.

NETZ, P. A.; ORTEGA, G. G. Fundamentos de físico-química: uma abordagem conceitual para as ciências farmacêuticas. Artmed, Porto Alegre, 2008. 299p.

SANTOS, D. Q.; VIEIRA, D. F.

Determinação de coeficiente de expansão térmica do biodiesel e seus impactos no sistema de medição volumétrico. Eclética Química, v. 35, n. 4, p. 107-112, 2010. 
SKOOG, D. A.; WEST, D. M.; HOLLER, F.

J.; CROUCH, S. R. Fundamentos de Química Analítica. Cengage Learning. São Paulo, 2006. 1124p.

TRIOLA, M. F. Introdução a Estatística.

Livros Técnicos e Científicos, Rio de Janeiro, 2008. 410p. 